\title{
DSS-28: A novel wide bandwidth radio telescope devoted to educational outreach
}

\author{
Glenn Jones ${ }^{a b}$, Sander Weinreb ${ }^{b c}$, Hamdi Mani ${ }^{b}$, Stephen Smith $^{b}$, Lawrence Teitelbaum ${ }^{c}$, \\ Mark Hofstadter ${ }^{c}$, Thomas B. H. Kuiper ${ }^{c}$, William A. Imbriale ${ }^{c}$, Ryan Dorcey ${ }^{d}$, and John \\ Leflang ${ }^{d}$ \\ ${ }^{a}$ Jansky Fellow, National Radio Astronomy Observatory, USA; \\ ${ }^{b}$ California Institute of Technology, 1200 E. California Blvd., Pasadena, CA, USA; \\ ${ }^{c}$ Jet Propulsion Laboratory 4800 Oak Grove Blvd., Pasadena, CA, USA; \\ ${ }^{d}$ Lewis Center for Educational Research 17500 Mana Road, Apple Valley, CA, USA;
}

\begin{abstract}
We have recently equipped the 34-meter DSS-28 radio telescope at the Goldstone Deep Space Communications Complex with a novel wide bandwidth radiometer and digital signal processor as part of the Goldstone Apple Valley Radio Telescope (GAVRT) educational outreach program operated by the Jet Propulsion Laboratory and the Lewis Center for Educational Research. The system employs a cryogenically cooled wide bandwidth quad-ridge feed and InP low noise amplifiers to achieve excellent noise performance from 2.7 to $14 \mathrm{GHz}$; a fractional bandwidth better than 4:1. Four independently tunable dual-polarization receivers each down-convert a $2 \mathrm{GHz}$ block to baseband, providing access to $8 \mathrm{GHz}$ of instantaneous bandwidth. A flexible FPGA-based signal processor has been constructed using CASPER FPGA hardware and tools to take advantage of this enormous bandwidth. This system demonstrates many of the enabling wide bandwidth technologies that will be crucial to maximizing the utility of future large centimeter-wavelength arrays, in particular the Square Kilometer Array. The GAVRT program has previously used narrow bandwidth total power radiometers to study flux variability of quasars and the outer planets. The versatility of DSS-28 will enable other projects including spectroscopy and SETI. Finally, the wide instantaneous bandwidth available makes this system uniquely suited for studying transient radio pulses. A configuration of the digital signal processor has been developed which provides the capability of recording a burst of raw baseband voltage data triggered by a real-time incoherent dedispersion system which is very sensitive to pulses from a known source, such as the Crab Nebula pulsar.
\end{abstract}

Keywords: Wide bandwidth, educational outreach, radio astronomy, pulsars, low noise amplifiers

\section{INTRODUCTION}

The reduced cost of electronics relative to steelwork is often used to argue that future radio telescopes should be implemented as large arrays of small, inexpensive dishes instead of traditional large dishes. Fully realizing this advantage, however, requires receivers with much broader bandwidths to reduce the total number of receivers which must be replicated across the array. In addition to the practical advantages, increased receiver bandwidth

Further author information: (Send correspondence to G.J.)

G.J.: E-mail: jones_gl@caltech.edu

S.W.: E-mail: sweinreb@caltech.edu

H.M.: E-mail: hamdi@caltech.edu

S.S.: E-mail: steves@caltech.edu

L.T.: E-mail: lawrence.teitelbaum@jpl.nasa.gov

M.H.: E-mail: mark.hofstadter@jpl.nasa.gov

T.B.H.K.: E-mail: kuiper@jpl.nasa.gov

W.A.I.: E-mail: william.a.imbriale@jpl.nasa.gov

R.D.: E-mail: rdorcey@lcer.edu

J.L.: E-mail: jleflang@pacbell.net

Ground-based and Airborne Telescopes III, edited by Larry M. Stepp, Roberto Gilmozzi, Helen J. Hall Proc. of SPIE Vol. 7733,773330 - (c) 2010 SPIE - CCC code: 0277-786X/10/\$18 - doi: 10.1117/12.856642 
can also provide unique opportunities to study otherwise inaccessible astrophysical phenomena. The DSS-28 system, described herein, demonstrates the practical and scientific advantages of a wide bandwidth receiver.

The 34 meter DSS-28 antenna dish, shown in figure 1(a), is located at the Goldstone Deep Space Communication Complex near Barstow, California. The antenna was mothballed for many years before it was offered to the Lewis Center for Educational Research (LCER)*. The LCER operates the Goldstone Apple Valley Radio Telescope (GAVRT) program which aims to enrich K-12 science education through radio astronomy. Since its inception, the GAVRT program has allowed students from all over the world to remotely control via the Internet the 34 meter DSS-12 telescope to perform radio astronomy observations. The LCER partners with radio astronomers with scientific projects which would benefit from the large amount of observing time available through GAVRT. The astronomer is responsible for helping to develop a curriculum around the observations, and also interacts with the students to help them understand the importance of the measurements they are helping to make. DSS-12 is equipped with a legacy total power receiver providing up to $50 \mathrm{MHz}$ of bandwidth at around 2 and $8 \mathrm{GHz}$. The observational campaigns have generally been limited to monitoring flux variations of quasars and the outer planets. The students measure these sources using cross scans through the source and by making raster scanned maps.

When DSS-28 was offered to the LCER, it was realized that the program could be greatly expanded by getting more astronomers involved if a more versatile receiver system were available. Instead of equipping DSS-28 with a traditional narrow band total power radiometer as available on the original antenna, DSS-12, it was proposed that a novel wide band radiometer could be constructed to fit the same budget, yet provide a decade of frequency coverage. A digital signal processing system was also proposed to take full advantage of the capabilities of this instrument. ${ }^{1}$ This system employs many novel techniques, from the optics through the digital back end.

\subsection{Optics}

DSS-28 was originally constructed as a high power transmitting antenna. ${ }^{2}$ To this end, the Cassegrain optics of this antenna were designed such that the beam does not come to focus until deep into the beam waveguide. As a fortunate consequence, the radiation is approximately a plane wave at the entrance to the beam waveguide at the vertex of the dish. This allows an offset paraboloid to be used to focus the beam onto a feed mounted to a frame on the surface of the dish. Moreover, this offset paraboloidal tertiary can be rotated to direct the beam onto one of many feeds which can be mounted around the vertex of the dish. Currently the system is equipped with two feeds, but in the future other feeds could be added to test their performance or to expand the capabilities of the system. The optics and feed system are shown in figure 1(b).

\subsection{Feeds}

As with any radio telescope, wider bandwidth feeds help maximize the capabilities of the instrument for a given budget. The premier radio observatories all use highly optimized feeds to get the maximum sensitivity because the scale of the project is so large that the incremental cost of another feed is relatively insignificant. In contrast, the limited budget of DSS-28 provided a strong incentive to use the widest bandwidth feeds practical. This is a very relevant constraint to future large arrays, where each feed must be replicated across hundreds or thousands of small dishes. As such, DSS-28 provides a unique opportunity to demonstrate the kind of wide band technology necessary for large arrays like the SKA. While the original hope was to cover a decade of bandwidth with a single feed, at the time of the design, the best choice in terms of maximizing performance given the budget was to use two feeds to cover roughly $0.5 \mathrm{GHz}$ to $4 \mathrm{GHz}$, called the Low Frequency Feed (LFF), and 2 to $14 \mathrm{GHz}$, called the High Frequency Feed (HFF).

\subsubsection{The High Frequency Feed}

The High Frequency Feed is based around a commercially available feed designed for antenna pattern measurements and manufactured by ETS-Lindgren. ${ }^{3}$ The beam pattern of the feed was measured at Caltech and found to have good performance. In particular, surrounding the feed with a metal can as a model of the Dewar and placing absorbing strips along the outer edge of the feed fins yielded a very constant beam width versus frequency. This is important to provide the best performance across frequency because a narrow beam will under-illuminate

\footnotetext{
*http://www.lcer.org
} 


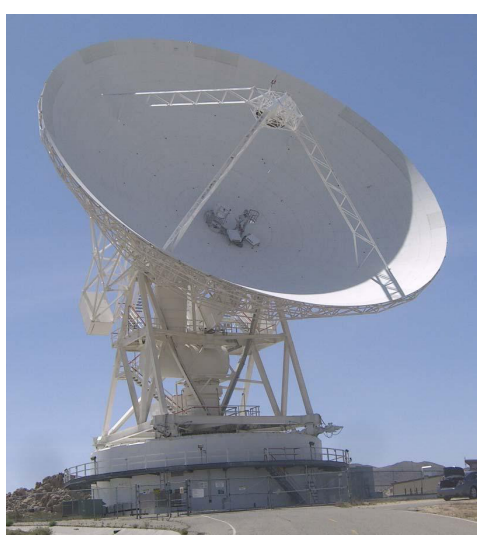

(a)

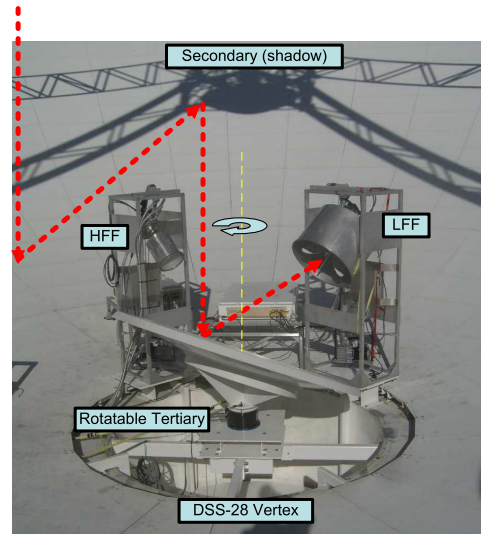

(b)

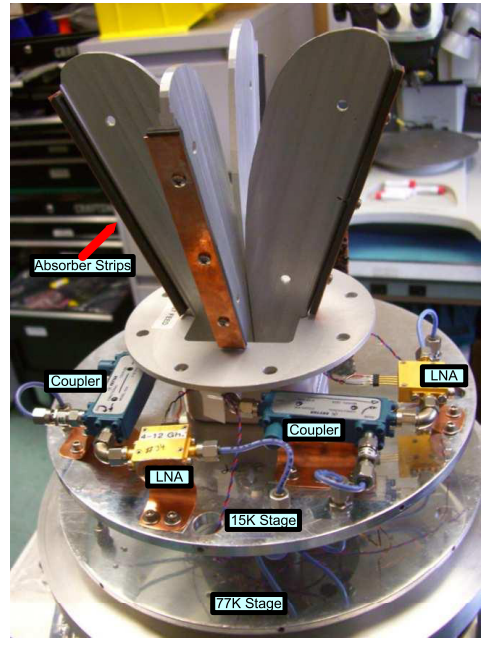

(c)

Figure 1. (a) The 34-meter DSS-28 antenna. The tertiary, feeds, and receiver are visible at the vertex. (b) The optical system as seen from the surface of the dish. The dashed red line shows the approximate path of an incoming ray. The dashed yellow line shows the axis of rotation of the tertiary. The box containing the receiver electronics can be seen in between the High and Low Frequency Feed support towers. The entrance of the original beam waveguide is also visible. (c) View of the High Frequency Feed without the surrounding cryogenic Dewar.

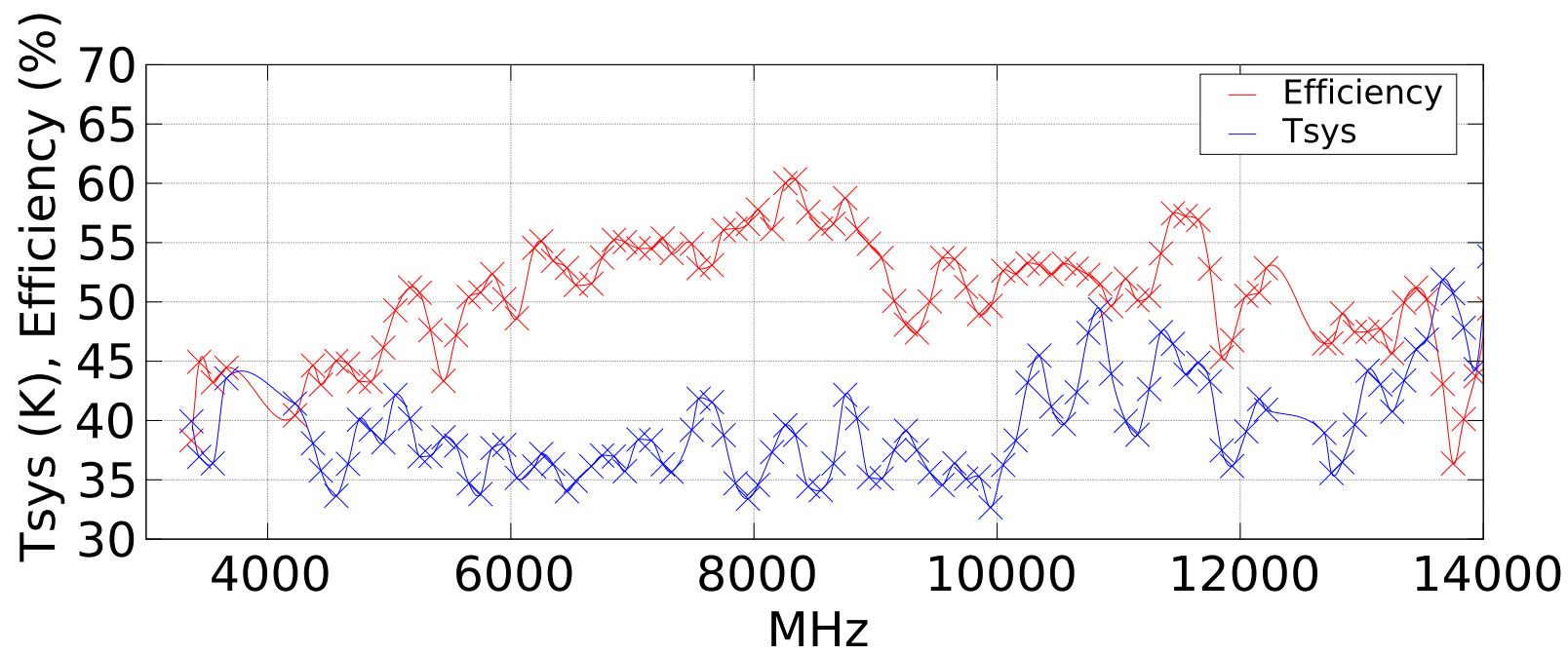

Figure 2. System temperature (lower curve) and aperture efficiency (upper curve) measurements of the HFF. The measured points are indicated by crosses. Interpolated curves have been added for clarity. 
the reflector, yielding reduced efficiency, while a wide beam will see more spillover radiation from the ground and degrade the system noise. ${ }^{4}$ For an uncooled feed, the absorbing strips would significantly impact the noise of the system, but fortunately the High Frequency Feed is physically small enough that it could be cryogenically cooled.

The HFF cryogenics package is shown in figure 1(c). The feed is mounted directly to the 12 kelvin stage of a standard CTI-350 cryogenic refrigerator. The output from each polarization is fed into a $30 \mathrm{~dB}$ coupler used to inject calibration signals into the LNA which immediately follows the coupler. The noise calibration diodes are located in the Dewar, mounted to the first stage of the refrigerator. This greatly improves the stability of the calibration signals when compared to the traditional method of housing the noise diodes in another box, connected by coax cables.

Designing a window for the HFF cryogenic Dewar proved to be a significant challenge. The window must be made large enough to avoid interfering with the pattern of the feed. This requires that the window material be sufficiently strong to withstand approximately 1200 pounds of atmospheric pressure, yet be transparent to microwaves. In other applications, a number of rigid materials could be used by applying an anti-reflective impedance matching coating to the window. However, such techniques are not readily applicable to feeds covering more than an octave of bandwidth. After trying a number of plastic films and foams, a 15 mil mylar film proved to be the best solution. The window was found to be largely transparent to infrared radiation, which significantly loaded the cold stage causing the temperature to rise. The window also became very cold itself and accumulated moisture and ice, degrading the system noise temperature. To solve this problem, a blanket made of layers of Teflon film and paper was placed between the feed and the window.

The performance of the HFF is shown in figure 2. These measurements were made by observing the radio galaxy $3 \mathrm{C} 123$, a commonly used calibration source, ${ }^{5}$ at an elevation of around 75 degrees. The system temperature shown is measured off source. The system provides excellent performance from 2.7 to $14 \mathrm{GHz}$, and is usable to $16 \mathrm{GHz}$.

\subsubsection{The Low Frequency Feed}

The LFF is also based around an ETS-Lindgren feed, which is essentially a scaled version of the HFF. Because of its significantly larger size, it was deemed impractical to cool the feed itself. However, the LNAs are cooled to 50 $\mathrm{K}$ in a small Dewar by a Stirling cycle refrigerator. These refrigerators require no maintenance and are extensively used by the mobile phone industry. The architecture of the LFF is exactly the same as that of the HFF, with a cooled LNA and coupler for calibration signals. During the design phase, the efficiency of the LFF was predicted by scaling the calculations of the HFF performance, made using measured data. Unfortunately, after measuring the performance of the LFF it was realized that secondary reflector on the telescope was undersized for the frequency range of the LFF. Physical optics calculations were then performed and found to agree with the observed linear decrease in efficiency with increasing wavelength. The aperture efficiency appears to decrease linearly from a peak of $35 \%$ at $1.7 \mathrm{GHz}$ to nearly $0 \%$ at $500 \mathrm{MHz}$. The system temperature is around $100 \pm 25 \mathrm{~K}$ across this band, improving to $75 \mathrm{~K}$ between 1.7 and $3 \mathrm{GHz}$.

Another problem with the LFF is the $50 \mathrm{~K}$ refrigerator is considerably less effective at cryopumping than the $12 \mathrm{~K}$ refrigerator, making the Dewar extremely sensitive to the effectiveness of the vacuum seal.

These issues, along with the large amount of RFI present in the lower frequency band make for an uncertain future for the LFF. It should be noted, however, that the LFF may still be quite effective for observing giant pulses from the Crab pulsar because the flux from the surrounding nebula is so great that it will largely dominate the system temperature despite the low efficiency.

\subsection{Tertiary Response}

To properly align the tertiary with the feed, the response of the system was measured versus tertiary angle. These measurements were performed on the methanol maser source $\mathrm{W} 3 \mathrm{OH} .{ }^{6}$ This source exhibits strong, narrow, spectral lines at 6.67 and $12.179 \mathrm{GHz}$. By measuring a spectral source, we can use the frequency channels away from the spectral line to measure the system noise simultaneously. The maser emission in W3OH is also extremely compact compared to the beamwidth of DSS-28, avoiding the need to apply source size corrections. ${ }^{7}$ Figure 3 


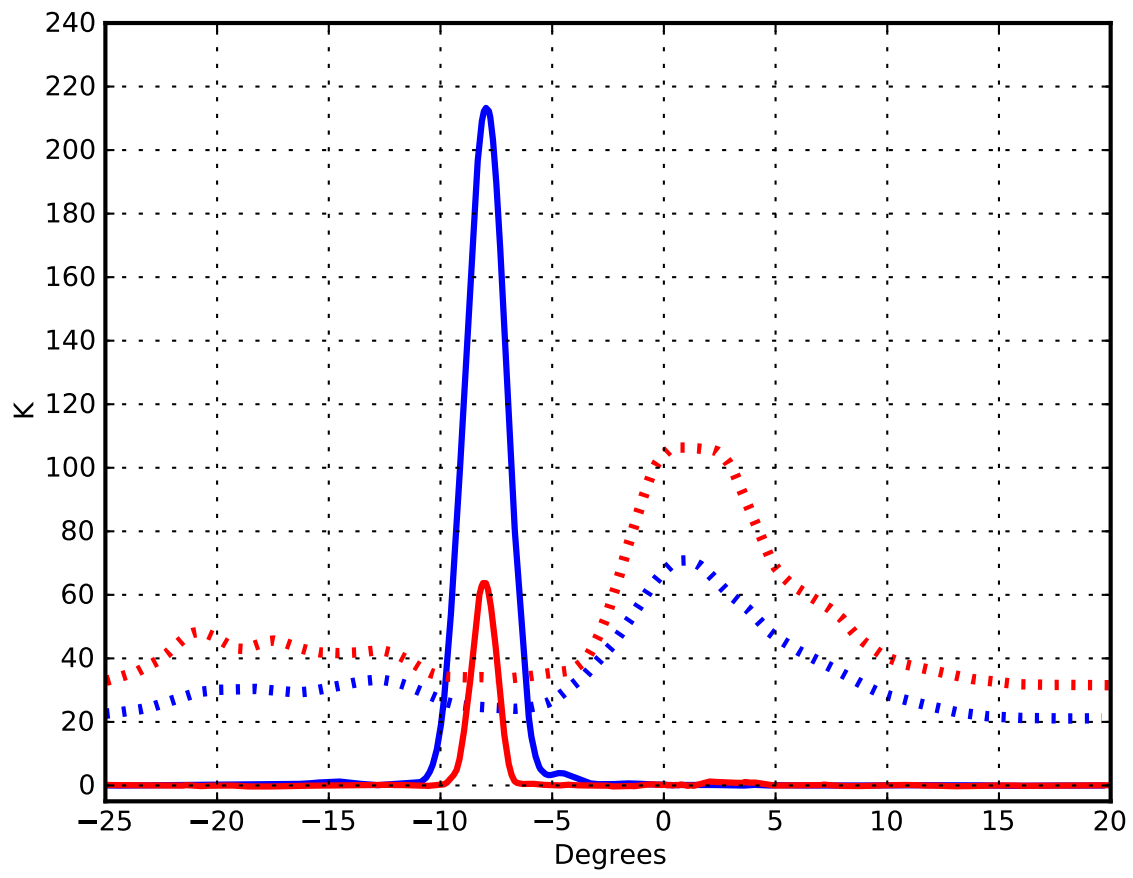

Figure 3. Measured response as a function of tertiary angle for the methanol maser lines in W3OH. The system temperatures are plotted as dotted lines while the antenna temperatures of the maser lines are solid. The 6.67 GHz measurements are plotted in blue (higher antenna temperature, lower system temperature), while the $12.179 \mathrm{GHz}$ measurements are in red (lower antenna temperature, lower system temperature).

shows the measured antenna temperature of the source and the system noise versus tertiary angle at both 6.67 and $12.179 \mathrm{GHz}$. The antenna temperature was calculated using the noise calibration diodes which were in turn calibrated using a Y-factor measurement between microwave absorber at ambient temperature and cold sky. ${ }^{8}$ The antenna temperature of the source was measured by subtracting the local spectral baseline. Two features are striking in the tertiary response. First, the system noise temperature has a local minimum near the optimal angle, increasing to either side. This increase is understood to be the result of increased spillover radiation being scattered off the secondary reflector support legs. The asymmetry from one side to the other is partly due to inherent asymmetry in the feed itself. The asymmetry may also be partly attributed to asymmetry in the location of the feed relative to the support legs. When the angle is far from optimal, the system noise drops below the local minimum at the optimal position. This is presumably because spillover radiation seen by the secondary is now no longer focused on the feed. Further measurements are needed to characterize this effect versus antenna azimuth and elevation position. The second striking feature is the asymmetry in the antenna temperature response, which exhibits a small shoulder on the positive side. This may be due to the same asymmetric geometry which causes the asymmetric system temperature response, but may also point to some residual misalignment in the optical system.

\subsection{Wide Bandwidth Low Noise Amplifiers}

The wide band cryogenic low noise amplifiers used for the High Frequency Feed are some of the most mature technology in the system. The amplifiers, designed by Weinreb and Wadefalk, are based around indium phosphide (InP) high electron mobility transistors (HEMTs), and have been the work horses of the radio astronomy and physics communities for the past ten years.

In general, amplifier design requires a trade-off between low noise and linearity at large signal levels. Radio astronomy applications demand amplifiers with the absolute lowest noise, which generally results in relatively poor linearity and therefore degradation in the presence of strong interfering signals. While HEMTs achieve by 


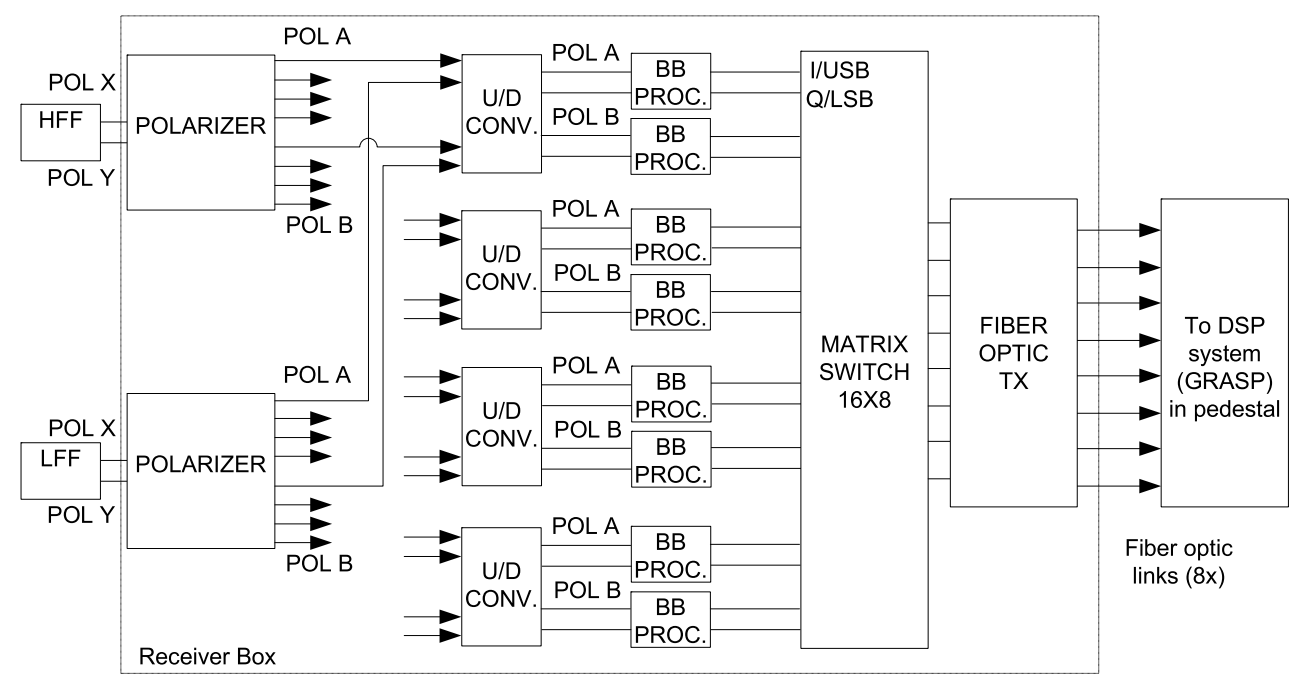

Figure 4. Block diagram of the receiver system.

and large the best noise performance, like any field effect transistor, they suffer from higher flicker noise compared to bipolar devices. This noise modulates the gain of the amplifier and degrades the stability on timescales of $\sim 1$ second for a $1 \mathrm{GHz}$ bandwidth observation. ${ }^{9,10}$

The LNA used for the LFF is a new design based around silicon germanium (SiGe) bipolar transistors. Recent work by ${ }^{11}$ has demonstrated cryogenic SiGe amplifiers with noise performance rivaling or exceeding that of HEMTs at lower microwave frequencies.

In narrow bandwidth applications, the deleterious effect of non-linearity on system performance is usually due to third-order intermodulation products which land within the band of interest, and thus pass through the intermediate frequency filters in a heterodyne receiver. With the wide bandwidth of the GAVRT system, it was found that the second-order intermodulation products are also of great concern, because the larger analyzed bandwidth will encompass them. Laboratory measurements of this effect were made with the SiGe LFF LNA and presented by. ${ }^{12}$ When a large interfering signal is present, the amplifier acts like a mixer with the large signal acting as the local oscillator.

\section{THE WIDE BANDWIDTH RECEIVER}

In keeping with the goal of maximizing the variety of science observations possible with DSS-28, a very flexible receiver was designed to take advantage of the unique wide bandwidth front ends. The receiver, shown in figure 4 , is composed of four independently tunable, dual-polarization receiver chains, each of which can down-convert a $2 \mathrm{GHz}$ band to baseband as a pair of $1 \mathrm{GHz}$ upper/lower sideband or in-phase/quadrature signals. The polarizer blocks provide the option of switching in a quadrature hybrid to provide circular polarization instead of the native linear polarization of the feeds. In each receiver chain, the up/down-converter block uses a tunable local oscillator from 22 to $38 \mathrm{GHz}$ to up-convert the RF signal to an IF signal centered around $22 \mathrm{GHz}$. The IF signal is filtered by either a $21-23 \mathrm{GHz}$ or a $22.1-22.5 \mathrm{GHz}$ waveguide filter. After filtering, the IF signal is down-converted to baseband with a quadrature mixer driven with a $22 \mathrm{GHz}$ local oscillator. The baseband processor blocks then take these signals and provide the option of using a quadrature hybrid for sideband separation, as well as level setting with a digital variable attenuator, and several filter options to select a final baseband bandwidth of 100 , 500, or $1000 \mathrm{MHz}$.

Unfortunately, the photonic modules necessary to bring the baseband signals down to the back end via optical fibers ended up being a major cost limitation. At the same time, this aspect of the system can be easily upgraded in the future to improve the capabilities of the system. As built, 8 fiber optic links are available, which allow 8 


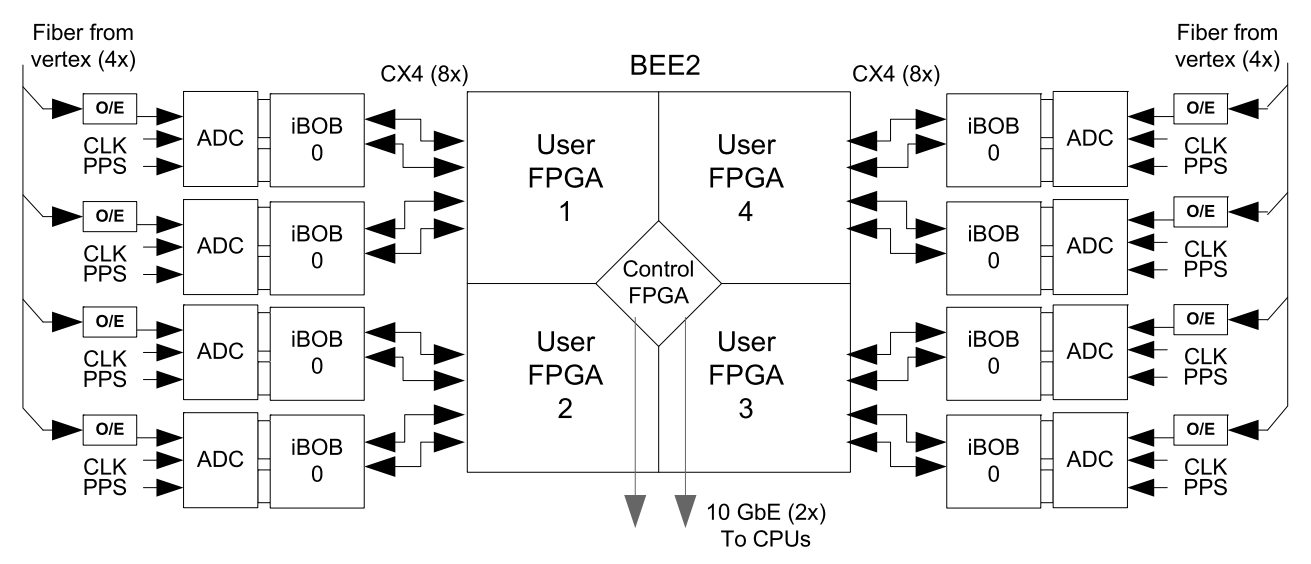

Figure 5. Block diagram of the digital signal processing system.

of the 16 baseband signals to be connected to the desired back end. A matrix switch is available in the receiver to allow any one of the 8 fibers to be connected to any one of the 16 baseband signals.

\section{THE DIGITAL SIGNAL PROCESSING SYSTEM}

While the GAVRT receiver was designed to be compatible with existing baseband spectrometers and signal recorders, including the Wide-band VLBI Science Recorder, none of the available systems would take full advantage of the enormous instantaneous bandwidth provided by the receiver. Therefore a new system was desired which would provide versatility and enable unique science to be performed with this instrument.

The primary goals of the digital signal processing subsystem were to make use of as much of the bandwidth available while meeting the tight cost requirements of the project. Many science campaigns were foreseen as desirable applications of the new radio telescope, each with different signal processing requirements. Therefore, the signal processor was required to be highly configurable. To best meet these goals, it was decided to implement the signal processor using field programmable gate arrays (FPGAs), as is becoming increasingly common in radio astronomy. The enormous capability and versatility of FPGAs comes at the cost of engineering complexity, which in the past has required such large design efforts that any given system is obsolete by the time it is complete. To help alleviate this problem, the Collaboration for Astronomical Signal Processing Equipment Research (CASPER) ${ }^{\dagger}$ group at the University of California, Berkeley, has built up a collection of open-source hardware and 'gateware' (firmware for FPGAs) specifically oriented towards radio astronomy applications. ${ }^{13}$ By standardizing on a set of boards, software tools, and libraries, they have effectively distributed the large non-recoverable engineering costs associated with complex FPGA-based signal processors.

Designing the GAVRT signal processor using the CASPER tools provided a far more versatile system at a much lower cost than would have been possible using closed-source, commercial solutions. Additionally, CASPER hardware has rapidly proliferated to observatories around the world which provides the benefit that signal processor designs developed at GAVRT can be used on other telescopes around the world, and similarly, designs originally made for other telescopes can be used at GAVRT. Inevitably, the digital hardware around which the GAVRT signal processor was built has already been superseded by the next generation of FPGA technology. Fortunately, new boards are already available from the CASPER group and it will be a relatively simple matter to port the designs described herein to the new hardware.

\subsection{Detailed architecture description}

The GAVRT Radio Astronomy Signal Processor (GRASP) is composed of digitizers, two types of interconnected FPGA boards, and a small cluster of PCs. The overall architecture is shown in figure 5

\footnotetext{
${ }^{\dagger}$ http://casper.berkeley.edu/
} 


\subsubsection{Digitizers}

The eight baseband outputs from the matrix switch are sent down to the pedestal room over fiber optic links. The output of each fiber optic receiver is connected to a CASPER digitizer board, called the iADC. This board contains an E2V (formerly Atmel) AT84AD001B ADC. Internally, this chip contains two ADCs which can sample at up to 1 GSps each. The ADCs can also be interleaved to provide an effective sampling rate of 2 GSps and be capable of digitizing a $1 \mathrm{GHz}$ bandwidth signal.

\subsubsection{The iBOB FPGA Interface Boards}

Each digitizer is connected to a board containing a single Xilinx Virtex II Pro FPGA, called an iBOB. The iBOB has sufficient resources to independently process the baseband signal. For example, for continuum observations, an $\mathrm{iBOB}$ can be configured with a polyphase filterbank spectrometer capable of dividing the band into 1024 channels. The low spectral leakage provided by the polyphase filterbank allows channels contaminated with RFI to be eliminated. The mean of the remaining uncorrupted channels then provides an equivalent total power output free of RFI. As another example, the iBOB can digitally down-convert a $64 \mathrm{MHz}$ band and then use a polyphase filterbank to break this band into 8192 channels for an effective spectral resolution of $8 \mathrm{kHz}$. This is sufficient resolution for some spectral line studies. One of the chief limitations in using an iBOB independently is that the maximum practical data rate through the $100 \mathrm{Mbit}$ Ethernet interface is only about $7 \mathrm{Mbit} / \mathrm{s}$, which is insufficient for most pulsar observations.

Fortunately, the iBOB provides two $10 \mathrm{Gbit} / \mathrm{s}$ CX-4 XAUI ports which provide ample I/O bandwidth. Each CX-4 port can be used as a point-to-point XAUI link, or as a standard $10 \mathrm{Gbit} / \mathrm{s}$ Ethernet (10 GbE) link. In GRASP, all of the iBOB CX-4 ports are used as point-to-point XAUI links because the FPGA resource requirements are much smaller than for $10 \mathrm{GbE}$. However, this could be upgraded in the future, which would provide dynamic connections between all of the FPGAs in the system through a commercially available $10 \mathrm{GbE}$ network switch, enabling a multitude of packetized signal processing architectures described by ${ }^{14}$

\subsubsection{The BEE2 FPGA Processing Board}

To provide more FPGA resources, the CASPER group has also developed the Berkeley Emulation Engine 2 (BEE2) board, which contains five interconnected Virtex II Pro FPGAs. ${ }^{15}$ The five FPGAs are arranged as four 'User' FPGAs and one 'Control' FPGA. The Control FPGA is able to reprogram each of the User FPGAs. The User FPGAs are connected in a ring and to the Control FPGA via digital I/O lines. One unique feature of the BEE2 compared to many commercial products which provide large numbers of interconnected FPGAs for computational acceleration is the I/O bandwidth provided. The BEE2 provides 18 CX-4 XAUI ports for 180 Gbit/s bidirectional I/O. These are allocated as 4 XAUI ports per User FPGA and 2 for the Control FPGA. Finally, each FPGA is connected to 4 DDR2 DRAM memory modules, providing up to a total of 20 Gbytes of DRAM.

In the GRASP design, each User FPGA is connected to two iBOBs via its 4 XAUI links. The two XAUI links from the Control FPGA are configured as $10 \mathrm{GbE}$ ports and connected to a commercial Ethernet switch which provides $241 \mathrm{GbE}$ ports to distribute the data flowing from the BEE2 to a small cluster of computers.

\subsubsection{Clocking and synchronization}

Many scientific goals and instrument designs require careful synchronization between the digitizers and accurate timestamps. Additionally, high speed digitization places stringent requirements on the sampling clock.

The DSS-28 system uses standard Deep Space Network Time Code Translator (TCT) modules as reference clocks and oscillators. The TCT is connected by fiber to the station maser from which it produces synchronous $100 \mathrm{MHz}$ and 1 pulse per second reference signals. The local oscillators in the DSS-28 wide bandwidth radiometer are all locked to this $100 \mathrm{MHz}$ reference.

The ADC sampling clocks will also be locked to the $100 \mathrm{MHz}$ reference. The sampling clocks are generated by Analog Devices AD9517 synthesizers which include an on chip integrated VCO, PLL, and dividers. They are capable of producing clock rates from $10 \mathrm{MHz}$ to $1100 \mathrm{MHz}$ with a gap between 750 and $880 \mathrm{MHz}$. The clock signals have excellent phase noise characteristics. This is important for high speed samplers because the sampling process is equivalent to multiplying by the sampling clock in the time domain which results in convolution of the spectrum of the sampled signal with the phase noise spectrum of the sampling clock. 


\section{SCIENCE APPLICATIONS}

The flexibility of the DSS-28 system will provide an improved instrument to support existing science campaigns, as well as enabling a wide range of new applications.

\subsection{Existing campaigns}

Over the past several years, three flux monitoring campaigns have been taking advantage of the large amount of observing time available with GAVRT. ${ }^{16}$

\subsubsection{Jupiter Quest}

Jupiter Quest has monitored synchrotron emission from Jupiter at 2.2 and $8.4 \mathrm{GHz}$ for many years. These measurements provide a means to study the interaction between the Jovian magnetosphere and the solar wind. The DSS-28 Low Frequency Feed will provide access to lower frequencies, where the synchrotron emission is stronger. Steve Levin is leading this study.

\subsubsection{Uranus Campaign}

This campaign uses flux measurements of Uranus to study the atmosphere of the planet, and in particular to look for seasonal variations. These measurements are used in conjunction with measurements made by many other observatories to gain a better understanding of the planet. The study is being lead by Mark Hofstadter.

\subsubsection{Quasar Variability Study}

This campaign, lead by David Jauncy of the Australia National Telescope Facility, measures the flux of several quasars known to exhibit intra-day variability. By measuring the flux at multiple frequencies, variability due to refractive interstellar scintillation can be separated from intrinsic variability in the quasar, leading to a better understanding of both phenomena. DSS-28 will provide flux measurements over a 4:1 frequency range, giving a detailed look at the frequency structure of the variability mechanisms.

\subsection{New campaigns}

\subsubsection{Giant pulses from the Crab nebula pulsar}

The details of the emission mechanism of radio pulsars remains poorly understood despite extensive research. One approach to gain insight into this problem is to study individual radio pulses in detail. This is difficult in general because individual pulses are extremely faint, and often undetectable even with the largest telescopes. A handful of pulsars, however, exhibit giant pulses which are more than 10 times brighter than average pulses. In particular, the Crab pulsar, at the heart of the Crab nebula, has been observed to emit pulses with fluxes greater than $2 \times 10^{6} \mathrm{Jy} .{ }^{17}$ These pulses occur across the radio spectrum, and have been observed from $23 \mathrm{MHz}$ to $25 \mathrm{GHz} .{ }^{18}$ Individual pulses extend over fractional bandwidths greater than $20 \% .{ }^{1719}$ Previous studies of Crab giant pulses have generally focused on statistics in a relatively narrow bandwidth at a single frequency, ${ }^{202122}$ or have studied individual pulses in great detail over the widest possible bandwidth. ${ }^{1719}$ The few observations that have been made using multiple telescopes to capture pulses at widely separated frequencies are limited to relatively narrow bandwidths at each frequency and are generally limited in observing time. ${ }^{231824}$

DSS-28 provides a unique opportunity to study individual giant pulses with up to $8 \mathrm{GHz}$ of instantaneous bandwidth, while simultaneously obtaining detailed statistics over hundreds of hours of observing time. The pulses follow a power law flux distribution, ${ }^{20}$ and have been detected at DSS-28 at a rate of a few per minute in preliminary observations, but this rate may increase with different detection configurations. As part of the GAVRT program, the observations will be made by participating students. The students will receive real time feedback when giant pulses are detected, in the form of a dynamic spectrum, and perhaps also an audio indication. They will quickly learn to distinguish genuine pulses from false positives caused by RFI, and will also learn about the different pulse morphologies. Some activities will be directly related to important scientific questions, for example, measuring the spectral index of a pulse or making a plot of the number of pulses observed versus their strength. Both of these examples provide practical examples of the importance of logarithms and exponentials. By combining data taken during one observation session with data taken by all students over the course of the campaign, students can also study the striking variability of the pulses over various timescales, which will provide 


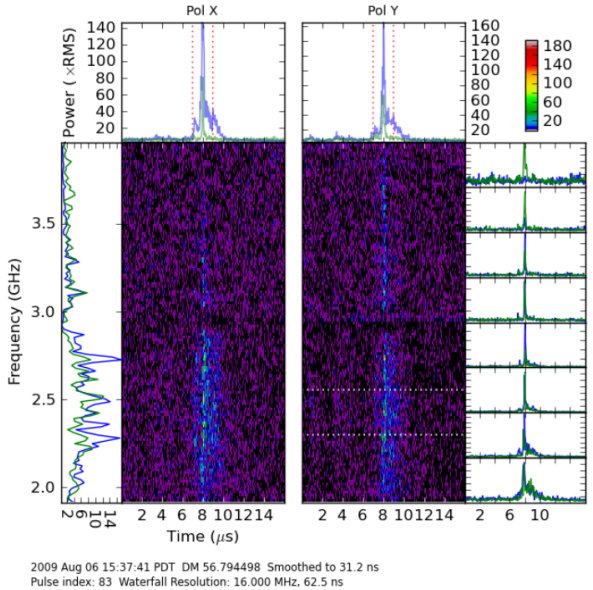

(a)

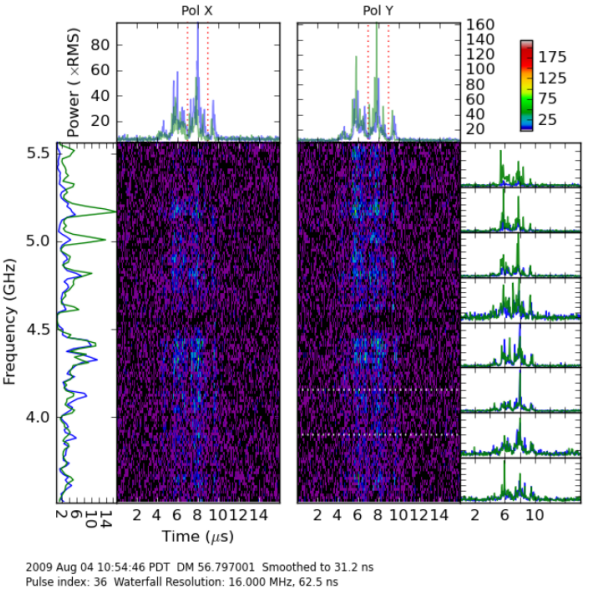

(b)

Figure 6. Individual giant pulses from the Crab pulsar captured with $2 \mathrm{GHz}$ total bandwidth using DSS-28. Each polarization and sideband was processed independently. In each example, the panel to the left of the dynamic spectra shows the spectrum averaged over the on pulse region indicated by the dotted vertical red lines in the top time domain plots. On the right, time series for each of eight equal subbands are plotted. The dotted white lines in the dynamic spectra plots show the frequency band used to trigger the instrument. ${ }^{1}$

insight into which features of the variability are intrinsic to the pulsar, and which are caused by propagation effects in the interstellar medium. Examples of giant pulses detected during early testing of the DSS-28 system are shown in figure 6.

In addition to studying the radio emission by itself, another aspect of the campaign will involve looking for correlations between radio giant pulse activity and gamma-ray emission detected by the Fermi satellite.

\subsubsection{Spectral line campaigns}

Table 1 is a list of common interstellar lines which fall within frequency range accessible to DSS-28 and might be used to develop research projects which would be appropriate for K-12 students. ${ }^{16}$ One might envision projects which involve extensive searches, such as ones not biased by a priori selection criteria, and long-term monitoring of maser emission, which is known to vary on timescales of weeks to months. DSS-28 would be particularly well

Table 1. Important spectral lines between 1.2 and $14.5 \mathrm{GHz}$.

\begin{tabular}{|r|c|c|l|}
\hline $\mathrm{GHz}$ & Species & Transition & Notes \\
\hline 1.420 & $\mathrm{H}$ & $(1,1)-(1,0)$ & \\
1.612 & $\mathrm{OH}$ & $1-2$ & stellar envelope probe \\
1.665 & $\mathrm{OH}$ & $1-1$ & stellar envelope probe \\
1.667 & $\mathrm{OH}$ & $2-2$ & stellar envelope probe \\
1.721 & $\mathrm{OH}$ & $2-1$ & stellar envelope probe \\
4.830 & formaldehyde & $1_{1,1}-1_{1,0}$ & density probe \\
14.488 & formaldehyde & $2_{1,1}-2_{1,2}$ & density probe \\
6.668 & methanol & $\mathrm{A}^{+} 5_{1}-6_{0}$ & Type II; radiatively excited \\
9.936 & methanol & $\mathrm{E}^{-} 9_{-1}-8_{-2}$ & Type I; collision. excited \\
12.179 & methanol & $\mathrm{E}^{-} 3_{-1}-2_{0}$ & Type II; radiatively excited \\
9.097 & HCCCN & $1,1-0,1$ & cold cloud core probe \\
9.098 & HCCCN & $1,2-0,1$ & cold cloud core probe \\
9.100 & HCCCN & $1,0-0,1$ & cold cloud core probe \\
11.119 & CCS & $2,1-1,0$ & cold cloud core Zeeman probe \\
\hline
\end{tabular}


suited to monitoring several spectral lines simultaneously, including maser lines of the same species. Another possibility might be extensive mapping, such as CCS in a starforming region like Taurus.

\subsubsection{Search for Extraterrestrial Intelligence}

SETI is a project that naturally engages students imaginations and benefits from the large amount of observing time available at DSS-28. The search will be carried out using a $\sim 130$ million channel spectrometer over 100 $\mathrm{MHz}$ of bandwidth to provide $\sim 1 \mathrm{~Hz}$ resolution. The spectrometer is built around an additional set of CASPER BEE2 and iBOB boards, and may be reprogrammed for other campaigns when SETI observations are not in progress. A racetrack scan pattern ${ }^{25}$ will be used to cover the sky efficiently while providing multiple passes to search for the expected Doppler shift of any detected signals. In addition, GRASP can be programmed to do a commensal blind search for dispersed impulsive transients.

\section{SUMMARY}

The combination of a sensitive, extremely bandwidth receiver and a flexible digital signal processing system makes DSS-28 a powerful instrument for studying a wide variety of scientific phenomena. In addition to continuing long-term flux monitoring projects of quasars and the outer planets, a number of new projects which will highlight the features of DSS-28 are underway, including studies of giant radio pulses, molecular masers, and SETI. It is hoped that the opportunity to pursue long term campaigns with this exciting instrument will further enrich the GAVRT educational outreach program by involving more scientists in the project.

\section{ACKNOWLEDGMENTS}

The authors would like to thank Xilinx, Inc. for their generous donation of FPGAs and software tools which were crucial to the development of the digital signal processor. The Crab gamma-ray/radio giant pulse study is partially supported by NASA through a grant from the Fermi Space Telescope guest observer program.

\section{REFERENCES}

1. G. Jones, Instrumentation for wide bandwidth radio astronomy. $\mathrm{PhD}$ thesis, California Institute of Technology, 2010.

2. W. A. Imbriale, "Design of a wideband radio telescope," in Proc. IEEE Aerospace Conference, pp. 1-12, Mar. 3-10, 2007.

3. V. Rodriguez, "An open-boundary quad-ridged guide horn antenna for use as a source in antenna pattern measurement anechoic chambers," IEEE Antennas and Propagation Magazine 48, pp. 157-160, Apr. 2006.

4. J. W. M. Baars, The Paraboloidal Reflector Antenna in Radio Astronomy and Communication, vol. 348 of Astrophysics and Space Science Library, Springer, 2007.

5. M. Ott, A. Witzel, A. Quirrenbach, T. P. Krichbaum, K. J. Standke, C. J. Schalinski, and C. A. Hummel, "An updated list of radio flux density calibrators," A\&A 284, pp. 331-339, Apr. 1994.

6. K. M. Menten, "The discovery of a new, very strong, and widespread interstellar methanol maser line," ApJ 380, pp. L75-L78, Oct. 1991.

7. V. Minier, J. E. Conway, and R. S. Booth, "VLBI observations of 6.7 and $12.2 \mathrm{GHz}$ methanol masers toward high mass star-forming regions. II. Tracing massive protostars," AEA 369, pp. 278-290, Apr. 2001.

8. J. D. Kraus, Radio astronomy, Cygnus-Quasar Books, 2 ed., 1986.

9. N. C. Jarosik, "Measurements of the low-frequency-gain fluctuations of a 30-ghz high-electron-mobilitytransistor cryogenic amplifier," IEEE Transactions on Microwave Theory and Techniques 44, pp. 193-197, Feb. 1996.

10. J. D. Gallego, I. Lopez-Fernandez, C. Diez, and A. Barcia, "Experimental results of gain fluctuations and noise in microwave low-noise cryogenic amplifiers," in Noise in Devices and Circuits II, F. Danneville, F. Bonani, M. J. Deen, and M. E. Levinshtein, eds., Noise in Devices and Circuits II 5470, pp. 402-413, SPIE, 2004.

11. S. Weinreb, J. C. Bardin, and H. Mani, "Design of cryogenic SiGe low-noise amplifiers," IEEE Transactions on Microwave Theory and Techniques 55, pp. 2306-2312, Nov. 2007. 
12. S. Weinreb, J. Bardin, H. Mani, and G. Jones, "Matched wideband low-noise amplifiers for radio astronomy," Review of Scientific Instruments 80, p. 044702 ff., Apr. 2009.

13. A. Parsons, D. Backer, C. Chang, D. Chapman, H. Chen, P. Crescini, C. de Jesus, C. Dick, P. Droz, D. MacMahon, K. Meder, J. Mock, V. Nagpal, B. Nikolic, A. Parsa, B. Richards, A. Siemion, J. Wawrzynek, D. Werthimer, and M. Wright, "Petaop/second FPGA signal processing for SETI and radio astronomy," in Proc. Fortieth Asilomar Conference on Signals, Systems and Computers ACSSC '06, pp. 2031-2035, Oct. 2006.

14. A. Parsons, D. Backer, A. Siemion, H. Chen, D. Werthimer, P. Droz, T. Filiba, J. Manley, P. McMahon, A. Parsa, D. MacMahon, and M. Wright, "A Scalable Correlator Architecture Based on Modular FPGA Hardware, Reuseable Gateware, and Data Packetization," PASP 120, pp. 1207-1221, Nov. 2008.

15. C. Chang, J. Wawrzynek, and R. W. Brodersen, "BEE2: a high-end reconfigurable computing system," IEEE Design Test of Computers 22, pp. 114-125, Mar. 2005.

16. T. B. H. Kuiper, M. D. Hofstadter, S. M. Levin, and D. MacLaren, "Interstellar molecules in K-12 education," in Revealing the Molecular Universe: One Antenna is Never Enough, D. C. Backer, J. M. Moran, \& J. L. Turner, ed., Astronomical Society of the Pacific Conference Series 356, pp. 261-+, Dec. 2006.

17. T. H. Hankins and J. A. Eilek, "Radio emission signatures in the Crab pulsar," ApJ 670, pp. 693-701, Nov. 2007.

18. M. V. Popov, A. D. Kuz'min, O. M. Ul'yanov, A. A. Deshpande, A. A. Ershov, V. V. Zakharenko, V. I. Kondrat'ev, S. V. Kostyuk, B. Y. Losovskiä, and V. A. Soglasnov, "Instantaneous radio spectra of giant pulses from the crab pulsar from decimeter to decameter wavelengths," Astronomy Reports 50, pp. 562-568, July 2006.

19. T. H. Hankins, J. S. Kern, J. C. Weatherall, and J. A. Eilek, "Nanosecond radio bursts from strong plasma turbulence in the Crab pulsar," Nature 422, pp. 141-143, Mar. 2003.

20. M. V. Popov and B. Stappers, "Statistical properties of giant pulses from the Crab pulsar," $A \& A A \mathbf{4 7 0}$, pp. 1003-1007, Aug. 2007.

21. N. D. R. Bhat, S. J. Tingay, and H. S. Knight, "Bright giant pulses from the Crab nebula pulsar: statistical properties, pulse broadening, and scattering due to the nebula," ApJ 676, pp. 1200-1209, Apr. 2008.

22. R. Karuppusamy, B. W. Stappers, and W. van Straten, "Giant pulses from the Crab pulsar: A wide-band study," ArXiv e-prints, Apr. 2010.

23. S. Sallmen, D. C. Backer, T. H. Hankins, D. Moffett, and S. Lundgren, "Simultaneous dual-frequency observations of giant pulses from the Crab pulsar," ApJ 517, pp. 460-471, May 1999.

24. J. M. Cordes, N. D. R. Bhat, T. H. Hankins, M. A. McLaughlin, and J. Kern, "The brightest pulses in the universe: Multifrequency observations of the crab pulsar's giant pulses," ApJ 612, pp. 375-388, Sept. 2004.

25. M. Klein, D. Burns, C. Foster, M. Garyantes, S. Gulkis, S. Levin, L. Olsen, H. Wilck, and G. Zimmerman, "The NASA SETI microwave observing project sky survey," in Third Decennial US-USSR Conference on SETI, G. S. Shostak, ed., Astronomical Society of the Pacific Conference Series 47, pp. 335-+, 1993. 